\title{
Simulation as a training modality
}

Simulation has become an increasingly important training modality in the training of our OB-GYN residents. There are number of reasons for this trend.

1) Patient safety. This is perhaps the most important reason for simulation training. It is no longer considered acceptable to let inexperienced doctors perform procedures on patients without some basic skills. The problem with "see one do, do one, teach one approach is multifold. One problem is it is difficult to standardize evaluation. Variation in patient comorbities makes this method difficult for evaluation. The degree to which residents can or should operate independently varies. Many of these skills can be practiced beforehand in a non threatening situation where mistakes can be made without harm to the patient. It also gives experienced residents a chance to practice situations that happen infrequently such as shoulder dystocia, so they are better prepared when the rare event occurs.

2) Work hour restrictions. A study in 2000 of two separate PGY 4 classes for the year immediately preceding and post work hour restriction implementation revealed significant decreases in resident experience in TAH, SUI procedures, and hysteroscopy. ${ }^{1}$ Today's residents have wider range of procedures to learn, more elaborate documentation, more presentations, etc and fewer hours to do this is in. Simulation training can help bridge the gap with fewer hours spent in the OR.

3) Increase in minimally invasive procedures. Laparoscopic, hysteroscopic and robotic procedures have increased dramatically over the past 20 years. These procedures require hand eye coordination that can be improved with simulation training. Advanced software can mimic more complex procedures. 
4) Improvement in communication and team dynamics in a group situation. Simulation can help improve communication between doctors, nursing and ancillary personal. It can also help define areas that need improvement.

At the University of lowa we have used a number of simulation modalities for our residents.

- Low fidelity simulationscircumcision, ovarian cystectomy, knot tying, episiotomy repair, $3^{\text {rd }}$ and $4^{\text {th }}$ degree lacerations, hysteroscopy and hysteroscopic resection of polyps and myomas

- Birth simulators- we have 2 birth simulators that we have used to practice normal spontaneous vaginal delivery, vacuum assisted delivery, forceps, breech delivery and shoulder dystocia.

- Laparoscopic box trainersovarian cystectomy, peg transfer, intracorporal and extracorporeal knot tying, pattern cutting, supracervical hysterectomy

- High fidelity models- essure tubal ligation

General surgery has already implemented a mandatory surgical simulation training program as requirement in resident training. As evidence mounts that surgical outcomes improve with preceding simulation training, it is just a matter of time that all surgical specialties will have this as requirement in their training program.

Gregory S. Skopec, MD Residency Skills Lab Director Department of Obstetrics and Gynecology University of lowa, lowa City, IA

\section{Reference}

1 Blanchard MH, Amini SB, Frank TM. Impact of work hour restrictions on resident case experience in an obstetrics and gynecology residency program. Am J Obstet Gynecol. $2004 \quad$ Nov;191(5):1746-51. PubMed PMID: 15547558. http://dx.doi.org/10.1016/j.ajog.2004.07.060 